Alter des tuffbildenden Gymnostomum curvirostrum an einem bestimmten Standort anf gegen $3000 \mathrm{Jahre}$, eine $\mathrm{Zahl}$, die natürlich weit davon entfernt ist, eine auch nur annäherungsweise exacte zu sein, da hierbei noch eine ganze Anzahl von Nebenumständen in Betracht kommen, Die Zeit, welche zur Bildung eines anderen Tufflagers, bei $\mathrm{St}$. Johann, in welchem $\mathrm{Hypnum}$ commutatum noch zu erkennen ist, erforderlich war, wird von Ung $\theta \mathbf{r}$ auf 5964 Jahre berechnet, Hypnum commutatum wächst jedoch viel rascher, als Gymnos tomum curvirostrum, das jährlich nur 3 Linien durchschnittlich ansetzt. Inmerhin mögen diese Zahlen dazu dienen, ein Beispiel für die fast unbegrenzte Vegetation perennirender Moose abzugeben.

Nach dieser Abhandlung beginnt eine Darstellung der "Bacillariaceen (Diatomaceen) " von Prof. Dr. E. Pfitzer (S. 403-414); mit dem Vorkommen und äusseren Ansehen dex Bacillariaceen anfangend und bis zu ihrem Baue fortgeführt, geschmückt mit vorzüglichen Abbildungen solcher Repräsentanten aus dem Kieselguhr von Eger und dem Tripelgestein von Richmond in Virginien.

A. Geheeb.

\title{
Annali di Chimica applicata alla Medicina, diretti da Pavesi e Schivardi. Milano.
}

Vor uns liegen einige Monatshefte dieser Zeitschrift für medicinische Chemie, deren Existenz wohl nur wenigen deutschen Collegen bekannt sein dürfte. Wenn gleich in erster Reihe für ein ärztliches Publikum bestimmt, bietet sie doch auch für den Apotheker einiges Interesse, wenigstens in ibrem "Farmacia " überschriebenen Abschnitt, vielleicht auch in demjenigen, welcher dio Ueberschrift "Terapentica" trägt. Werden doch auch in unserer deutsehen pharmaceutischen Fachpresse therapeutische Notizen von Jahr zu Jahr häufger und wohl meist nicht ungern gesehen. Allerdings haben solche für den $A$ potheker in Ländern romaniseher Zunge weit höheren Werth, weil dort die Consultation des Apothekers in leichten Erkrankungen nicht wie bei uns einen verpönten Abusus, sondern die selbstverständliche Regel bildet.

Von pharmaceutischem Interesse ist in den vorliegenden Heften zunächst ein Vorschlag von Ricci: Sulla conservazione degli estratti mediante la glicerina. Unter voller Würdigung der von Duquesnel eingeführten Bereitungsweise der Extracte mit Glycerin, welche im fertigen Zustande 50 Procent ibres Gewichtes Glycerin enthalten, glaubt der Verfasser doch einem einfachen Glycerinzusatz vol 10 Procent den Vorzug geben zu müssen, weil diese Menge zur Conservirung der Extracte und dauernden Erhaltung ihrer geschmeidigen Consistenz hinreicht, die dort nothwendige Verdoppelung der zu reichenden Dosis hier wegfällt, ausserdem auch bei der Verwendung zu Pillen kein zu grosses Volumen der letzteren erforderlich wird.

Einige sich anschliessende Vorschriften zum Ueberziehen von Pillen mit Gelatine oder auch mit Tolubalsam sind einem amerikanischen Journal, die Betrachtungen über die fleisehfressenden Pflanzen im Allgemeinen und über Carica Papaya im Besonderen von Dr. Gatti der "Gazzetta degli Ospedali" entnommen, wie denn überhaupt Originalarbeiten der seltenere Fall in der genannten Zeitsehrift sind. In der an zweiter Stelle erwähnten Abhandlung constatirt Gatti, dass ihm Auszüge von Carica Papaya bei Behandlung chronischer Diarrhöe von säuglingen die besten Dienste geleistet haben. Wenn er ferner bemerkt, es sei wahrscheinlich, dass Carica Papaya ein energiseh wirkendes und leicht isolirbares Verdauungsferment enthalte, so können wir hinzufügen, dass wir dieses Ferment im reinsten Zustande als von Merck dargestelltes Papayotin jüngst in den Händen gehabt und uns durch den Versuch überzeugt haben, dass dasselbe im Stande ist, von zwischen Filtrirpapier getrocknetem Blutfibrin sein $\mathrm{z}$ weihundertfaches Gewicht binnen wenigen Stunden zu verdauen. 
Weiterhin belehrt uns Limousin, wie schwierig es für den Laien sei, sich in kunstgerechter Weise einen Aufguss von irgendwelchen Vegetabilien zu bereiten, und dass er desshalb die "Sacearo-Tisana", den Zuekerthee oder besser Theezucker erfunden habe, denn so nennt er den in feste Form gebrachten Verdampfungsrückstand von Ausz ügen medicinischer Vegetabilien, in denen Zucker gelöst wurde. Ein Stück solchen "Kräuterzuckers", wie wir das Präparat schlichter nennen würden, soll dann durch Lösen in Wasser einen tadellosen "Thee" geben. Eine Vorschrift zu einem schön grünen Unguentum Populi zeigt, wie bei uns veraltete Mittel in anderen Ländern noch gehegt und gepflegt werden.

Aus der Zeitschrift „La Farmacia" ist eine Mittheilung von Plevani über einen Apparat zur Bestimmung des Bleiweisses in der Bleiglätte entlehnt. Wer je einmal eine Kohlensäurebestimmung im Marmor durch Ermittelung des Gewichtsverlustes nach dem Austreiben der Kohlensäure durch Chlorwasserstoff gemacht hat, wird aus der Besehreibung mit Vergniigen einen alten Bekannten erkennen. Natürlich wird statt der Salzsäure in diesem Falle Salpetersäure benutzt.

Eine Abkoohung der Blätter von Nerium Oleandor scheint in Italien häufig zur Erzielung eines Abortus benutzt zu werden, wenigstens begegnen wir in der Zeitschrift einer Abhandlung über die Reactionen des Oleandrins, welche mit der Erklärung des Verfassers $\mathrm{F}$ ino cehi schliesst, dass nach dem heutigen Stande der chemischen Wissenschaft es kein Mittel gebe, in den Eingeweiden einer in Folge des Genusses von Oleanderabkochung zu Grunde gegangenen Frau mit Sicherheit den Gebrauch dieses Abortivmittels nachzuweisen, weil die Reactionen des Oleandrins eben in allen Punkten mit denjenigen eines der Selmi'schen Ptomaïne übereinstimmen.

Vom toxikologischen auf das therapeutisehe Gebiet übergehend, stossen wir auf eine Aeusserung Fenoglio's über das „Fer Bravais", welches er bei der Behandlung der Bleichsucht zwar nicht, wie Bertholet es behauptet, gänzlich wirkungslos, aber doch weit weniger wirksam gefunden hat, als Blaud'sche Pillen oder Eisenlactat. Die stattgehabte Wirkung auf den Organismus wurde nach einer exacten Methode, nämlich durch Bestimmung der Hämoglobinzunahme im Blut nachgewiesen.

Weder die Empfehlung von Jod oformbougies zur Behandlung der Gonorrhöe, noch diejenige des $\mathrm{Ch}$ inintannats in der Kinderpraxis wegen seiner Gesehmacklosigkeit, oder des Bromkaliums bei Blasenkatarrh, der mit salicylsaurem Natron getränkten Compressen bei Gelenkentzündungen, der Glycerineinreibungen bei Blattern, des Pilocarpins gegen Nachtsehweisse, noch ondlich die Beschreibung der Gefahren eines fortgesetzten Chloralgebrauches bieten für uns etwas Neues, und wenn unter der Rubrik "Chemica industriale " die amerikanische Erfindung des Celluloids, bekanntlich aus Pyroxylin und Campher hergestellt, besprochen, Vorschriften zu einem Cautschukkitt und zu Opodeldoc mitgetheilt werden, so interessirt uns vielleicht hiervon nur die in letzterem Falle angegebene Entfärbung mit Thierkohle, dagegen finden wir in dem der allgemeinen Chemie gewidmeten Abschnitt eine interessante Studie von Paterno iber eine Reihe von Fluorsubstitutionsproclucten, speciell über die drei von der Theorie vorausgesehenen Fluorbenzo ës äuren.

Wenn wir noch hinzufügen, dass die Absehnitte "Varietà", "Notizie " und "Rivista bibliografica" mit dem für sie passenden Material ausgestattet sind, so glauben wir den Charakter der Eingangs genannten Zeitschrift hinreichend skizzirt zu haben.

Heidelberg, im Februar 1882.

Dr. Vulpius. 